High Order Finite Volume Nonlinear Schemes for the Boltzmann Transport Equation

B. L. Bihari, P. N. Brown

March 31, 2005

Computational Methods in Transport Tahoe City, CA, United States September 11, 2004 through September 16, 2004 
This document was prepared as an account of work sponsored by an agency of the United States Government. Neither the United States Government nor the University of California nor any of their employees, makes any warranty, express or implied, or assumes any legal liability or responsibility for the accuracy, completeness, or usefulness of any information, apparatus, product, or process disclosed, or represents that its use would not infringe privately owned rights. Reference herein to any specific commercial product, process, or service by trade name, trademark, manufacturer, or otherwise, does not necessarily constitute or imply its endorsement, recommendation, or favoring by the United States Government or the University of California. The views and opinions of authors expressed herein do not necessarily state or reflect those of the United States Government or the University of California, and shall not be used for advertising or product endorsement purposes. 


\title{
HIGH ORDER FINITE VOLUME NONLINEAR SCHEMES FOR THE BOLTZMANN TRANSPORT EQUATION*
}

\author{
BARNA L. BIHARI AND PETER N. BROWN ${ }^{\dagger}$
}

March 29, 2005

\begin{abstract}
We apply the nonlinear WENO (Weighted Essentially Nonoscillatory) scheme to the spatial discretization of the Boltzmann Transport Equation modeling linear particle transport. The method is a finite volume scheme which ensures not only conservation, but also provides for a more natural handling of boundary conditions, material properties and source terms, as well as an easier parallel implementation and post processing. It is nonlinear in the sense that the stencil depends on the solution at each time step or iteration level. By biasing the gradient calculation towards the stencil with smaller derivatives, the scheme eliminates the Gibb's phenomenon with oscillations of size $O(1)$ and recudes them to $O\left(h^{r}\right)$, where $h$ is the mesh size and $r$ is the order of accuracy. Our current implementation is three- dimensional, generalized for unequally spaced meshes, fully parallelized, and up to fifth order accurate ("WENO5") in space. For unsteady problems, the resulting nonlinear spatial discretization yields a set of ODE's in time, which in turn is solved via high order implicit time-stepping with error control. For the steady-state case, we need to solve the non-linear system, typically by Newton-Krylov iterations. There are several numerical examples presented to demonstrate the accuracy, non-oscillatory nature and efficiency of these high order methods, in comparison with other fixed-stencil schemes.
\end{abstract}

1. Introduction. In the deterministic modeling of uncharged particle transport, physical processes are described by the Boltzmann transport equation (BTE) which is a linear integro-differential equation to be solved for the scalar unknown $\Psi$, usually called the particle flux. During the solution process, iterative methods are routinely used to solve the large systems of equations resulting from various discretizations, especially when it comes to solving the steady-state problem. As noted in [1], such approaches can be viewed as iterative solutions of a matrix equation

$$
A x=b
$$

for the unknown vector $x$ representing scalar fluxes $\Psi$, energy densities, or other integrated quantities of interest.

The spatial discretization has been traditionally done by some fixed stencil finite difference or finite element method typically solving for point values of the solution, thereby resulting in a "node-centered" scheme.

Finite volume schemes, on the other hand, were invented for the numerical solution of high speed fluid dynamics where conservation is crucial. Since they solve for the cell average of the unknown instead of its point values, they are sometimes refered to as "cell-centered" schemes as well. Application of these schemes to the BTE ensures not only conservation, but also provides for a more natural handling of boundary conditions, material properties and source terms, as well as an easier parallel implementation and post processing. The finite volume scheme also lends itself to an efficient implementation of high order spatial discretizations.

Material interfaces and time-dependent large source terms can introduce severe oscillations even with second order fixed stencil schemes. Slope limiting, or essentially nonoscillatory (ENO) spatial interpolations eliminate these oscillations, and make higher-than-second-order spatial accuracies possible. A newer variation of these nonlinear schemes is the Weighted ENO (WENO) scheme that makes the stencil transition less abrupt and boosts the accuracy in smooth regions. For unsteady problems, the resulting nonlinear spatial discretization yields a set of ODE's in time, which in turn is solved via high order implicit time-stepping with error control. For the steady-state case, we need to solve the non-linear system, typically by Newton-Krylov iterations. Both of these approaches would ideally expect a preconditioner in order to obtain a reasonable rate of convergence.

We will discuss the advantages of using an ENO/WENO method, as well as the various issues introduced by such nonlinear methods originally designed for computing shocked fluid flows. There will be several 1-D, 2-D, and

* This work was performed under the auspices of the U.S. Department of Energy by the Lawrence Livermore National Laboratory under contract W-7405-Eng-48.

${ }^{\dagger}$ Center for Applied Scientific Computing, Lawrence Livermore National Laboratory, Livermore, California 94550. 
3-D numerical examples presented to demonstrate the accuracy, non-oscillatory nature and efficiency of these high order methods, in comparison with other fixed-stencil schemes.

The paper is organized as follows. In $\S 2$, we describe the continuous problem from which the discrete linear system (1.1) is derived. In $\S 3$, the exact form of (1.1) is established via the introduction of the discrete ordinate angular discretization together with the nonlinear spatial discretization. Although these discretizations are described elsewhere in the literature, we present them in some detail for the sake of clarity and completeness. In $\S 4$ we show several numerical results, and close with concluding remarks in $\S 5$.

2. Background. In this section we first introduce the linear time-dependent BTE in three dimensional box geometry with general scattering [2]. The spatial domain is the box $\mathcal{D} \equiv\left\{r=(x, y, z) \mid a_{x} \leq x \leq b_{x}, a_{y} \leq y \leq\right.$ $b_{y}$, and $\left.a_{z} \leq z \leq b_{z}\right\}$, the direction variable is $\Omega \in \mathcal{S}^{2}$, the unit sphere in $\mathbf{R}^{3}$, the energy variable is $E \in(0, \infty)$, time is $t$, and the equation in the flux $\psi=\psi(r, \Omega, E, t)$ is given by

$$
\begin{aligned}
& \frac{1}{v(E)} \frac{\partial \psi}{\partial t}(r, \Omega, E, t)+\Omega \cdot \nabla \psi(r, \Omega, E, t)+\sigma(r, E) \psi(r, \Omega, E, t)= \\
& \int_{0}^{\infty} \int_{\mathcal{S}^{2}} \psi\left(r, \Omega^{\prime}, E^{\prime}, t\right) \sigma_{s}\left(r, \Omega \cdot \Omega^{\prime}, E^{\prime} \rightarrow E\right) d \Omega^{\prime} d E^{\prime}+q(r, \Omega, E, t),
\end{aligned}
$$

with initial condition

$$
\psi\left(r, \Omega, E, t_{0}\right)=\psi^{0}(r, \Omega, E)
$$

where $\nabla \psi \equiv(\partial \psi / \partial x, \partial \psi / \partial y, \partial \psi / \partial z), v(E)$ is the particle speed, and $\psi^{0}$ is the initial state at time $t=t_{0}$.

The code employs a general algorithm that solves for multiple energy groups via a semi-discretization using a finite number of energy "bins". However, for simplicity of notation and clarity of exposition we shall assume a single energy group $E$ for the rest of this paper, and thereby remove the energy-dependency from $\psi, v, \sigma$ and $q$ in (2.1).

The scattering integral on the right hand side of (2.1) is handled by expanding the flux $\psi(r, \Omega, t)$ in surface harmonics according to

$$
\psi(r, \Omega)=\sum_{n=0}^{\infty} \sum_{m=-n}^{n} \phi_{n}^{m}(r, t) Y_{n}^{m}(\Omega) .
$$

Here, $Y_{n}^{m}(\Omega)$ is a surface harmonic defined by

$$
Y_{n}^{m}(\Omega)=a_{n}^{m} P_{n}^{|m|}(\xi) \tau_{m}(\varphi)
$$

where $\Omega=(\mu, \eta, \xi)=(\sin \theta \cos \varphi, \sin \theta \sin \varphi, \cos \theta), P_{n}^{|m|}$ is an associated Legendre polynomial [3], and

$$
\tau_{m}(\varphi)=\left\{\begin{array}{l}
\cos m \varphi, \text { if } m \geq 0, \text { and } \\
\sin |m| \varphi, \text { if } m<0
\end{array}\right.
$$

The constants $a_{n}^{m}$ are defined by

$$
a_{n}^{m}=\left[\frac{(2 n+1)(n-|m|) !}{2\left(1+\delta_{m 0}\right) \pi(n+|m|) !}\right]^{1 / 2}
$$

where $\delta_{n, n^{\prime}}$ is the Kronecker delta, and

$$
\phi_{n}^{m}(r) \equiv \int_{\mathcal{S}^{2}} \psi(r, \Omega) Y_{n}^{m}(\Omega) d \Omega,
$$

is the $(n, m)^{t h}$ moment of $\psi$. Similarly, the source $q$ is expanded as

$$
q(r, \Omega, t)=\sum_{n=0}^{\infty} \sum_{m=-n}^{n} q_{n}^{m}(r, t) Y_{n}^{m}(\Omega),
$$


where

$$
q_{n}^{m}(r, t) \equiv \int_{\mathcal{S}^{2}} q(r, \Omega, t) Y_{n}^{m}(\Omega) d \Omega
$$

For ease of exposition in what follows, we have elected to use real-valued surface harmonics, all scaled to have unit norm in $L^{2}\left(\mathcal{S}^{2}\right)$.

Given $\psi$ in the above form, one is able to rewrite the scattering integral in the form

$$
\int_{\mathcal{S}^{2}} \sigma_{s}\left(r, \Omega \cdot \Omega^{\prime}\right) \psi\left(r, \Omega^{\prime}, t\right) d \Omega^{\prime}=\sum_{n=0}^{\infty} \sigma_{s, n}(r) \sum_{m=-n}^{n} \phi_{n}^{m}(r, t) Y_{n}^{m}(\Omega)
$$

where the $\sigma_{s, n}$ are given by

$$
\sigma_{s, n}(r) \equiv 2 \pi \int_{-1}^{1} \sigma_{s}\left(r, \mu_{0}\right) P_{n}\left(\mu_{0}\right) d \mu_{0}
$$

and where $\mu_{0}$ is the cosine of the scattering angle. The total cross section $\sigma$ is given by

$$
\sigma(r) \equiv \sigma_{a}(r)+2 \pi \int_{-1}^{1} \sigma_{s}\left(r, \mu_{0}\right) d \mu_{0}=\sigma_{a}(r)+\sigma_{s, 0}(r)
$$

where $\sigma_{a}$ is the absorption cross section.

Substituting the appropriate terms into (2.1), the equation to be solved now becomes:

$$
\begin{aligned}
& \frac{1}{v} \frac{\partial \psi}{\partial t}(r, \Omega, t)+\Omega \cdot \nabla \psi(r, \Omega, t)+\sigma(r) \psi(r, \Omega, t)= \\
& \sum_{n=0}^{\infty} \sigma_{s, n}(r) \sum_{m=-n}^{n} \phi_{n}^{m}(r, t) Y_{n}^{m}(\Omega)+q(r, \Omega, t),
\end{aligned}
$$

Boundary conditions must also be specified so as to make (2.3) well-posed. Various options include a reflecting condition on a face, or a Dirichlet condition in which the incident flux is specified on a face. For simplicity, we will consider only the latter case. Namely, we will consider boundary conditions of the form

$$
\psi(r, \Omega)=g(r, \Omega) \text { for all } r \in \partial \mathcal{D} \text { and } \Omega \in \mathcal{S}^{2} \text { with } \vec{n}(r) \cdot \Omega<0
$$

where $\vec{n}(r)$ is the outward pointing unit normal at $r \in \partial \mathcal{D}$.

3. Discretization of the 3-D Problem. We now turn to the angular, spatial and temporal discretizations of (2.3). In previous work [4], we derived a matrix version of the well-known diamond difference discretization scheme for the 1-D slab problem analogous to (2.3)-(2.4). We extend that development here to 3-D problems. The first subsection deals with the quadrature rules for approximating integrals on $\mathcal{S}^{2}$, the next describes the spatial discretization, and the final subsection considers the discrete-ordinates method, i.e., the combined discretization of problem (2.3)-(2.4).

3.1. Quadrature Rules. The quadrature rules to approximate integrals on $\mathcal{S}^{2}$ use the standard symmetry assumptions. Following Carlson and Lathrop [5], the quadrature rules we consider are of the form

$$
\int_{\mathcal{S}^{2}} \psi(\Omega) d \Omega \approx \sum_{\ell=1}^{L} w_{\ell} \psi\left(\Omega_{\ell}\right)
$$

where $\Omega_{\ell} \equiv\left(\mu_{\ell}, \eta_{\ell}, \xi_{\ell}\right)$, for all $\ell=1, \ldots, L$, with $L=\nu(\nu+2)$ and $\nu$ is the number of direction cosines $(\nu=2,4,6, \ldots)$. Since $\Omega_{\ell} \in \mathcal{S}^{2}$ for all $\ell$, we have

$$
\mu_{\ell}^{2}+\eta_{\ell}^{2}+\xi_{\ell}^{2}=1 \text { for all } \ell
$$


With full symmetry, latitudinal point arrangement, and $\nu$ direction cosines, (3.2) becomes

$$
\xi_{i}^{2}+\xi_{j}^{2}+\xi_{\nu / 2+2-i-j}^{2}=1
$$

for $i=1,2, \ldots, \nu / 2$ and $j=1,2, \ldots, \nu / 2-i+1$. This last equation can be solved to give

$$
\xi_{i}^{2}=\xi_{1}^{2}+(i-1) \frac{2\left(1-3 \xi_{1}^{2}\right)}{\nu-2}
$$

for $i=1, \ldots, \nu / 2$ and $0<\xi_{1}^{2} \leq 1 / 3$.

For the weights, first note that a constant function on $\mathcal{S}^{2}$ must be integrated exactly for all $\nu$, and so we must have

$$
4 \pi=\int_{\mathcal{S}^{2}} 1 \cdot d \Omega=\sum_{\ell=1}^{L} w_{\ell}
$$

For $\nu=2$ or 4 , by requiring that weights be invariant under $90^{\circ}$ rotations of the $\Omega$ coordinate system, it is easily seen that each weight must be the same. For $\nu>4$ there are $\nu / 2$ distinct weights, and for these sets one can determine the weights by requiring that

$$
\sum_{\ell=1}^{L} w_{\ell} \xi_{\ell}^{2 n}=\frac{4 \pi}{2 n+1}
$$

for $n=0, \ldots, \nu / 2-1$. These conditions guarantee that as many even powers of $\xi$ as possible are integrated exactly by the quadrature rule. Note that due to the symmetrical placement of the $\xi_{\ell}$ along the $\xi$ axis, all odd powers of $\xi$ are integrated exactly. For $\nu \geq 22$, demanding that (3.4) holds for all $n=0, \ldots, \nu / 2-1$ leads to negative weights. As an alternative, one could simply demand that the weights are all equal, in which case (3.4) always holds for $n=0$ and 1.

For either type of quadrature rule, we only require that (3.4) holds with $n=0$ and 1 , and that all the weights are positive. Finally, it also follows from the symmetrical placement of the direction cosines that the following additional results hold:

$$
\sum_{\ell=1}^{L} w_{\ell} \mu_{\ell}=0, \sum_{\ell=1}^{L} w_{\ell} \eta_{\ell}=0, \text { and } \sum_{\ell=1}^{L} w_{\ell} \xi_{\ell}=0
$$

3.2. Finite Volume Spatial Discretization. In the formulation of the finite volume method, we first discretize $\mathcal{D}$ into cells (also called "zones"). Introduce the spatial grids

$$
\begin{aligned}
& a_{x} \equiv x_{\frac{1}{2}}<\cdots<x_{i-\frac{1}{2}}<x_{i+\frac{1}{2}}<\cdots<x_{M+\frac{1}{2}} \equiv b_{x}, \\
& a_{y} \equiv y_{\frac{1}{2}}<\cdots<y_{j-\frac{1}{2}}<y_{j+\frac{1}{2}}<\cdots<y_{J+\frac{1}{2}} \equiv b_{y}, \text { and } \\
& a_{z} \equiv z_{\frac{1}{2}}<\cdots<z_{k-\frac{1}{2}}<z_{k+\frac{1}{2}}<\cdots<z_{K+\frac{1}{2}} \equiv b_{z},
\end{aligned}
$$

and define $r_{i j k}=\left(x_{i}, y_{j}, z_{k}\right)$. Next, define

$$
\begin{aligned}
& \Delta x_{i}=x_{i-\frac{1}{2}}-x_{i+\frac{1}{2}} \text { for } i=1, \ldots, M \\
& \Delta y_{j}=y_{j-\frac{1}{2}}-y_{j+\frac{1}{2}} \text { for } j=1, \ldots, J, \text { and } \\
& \Delta z_{k}=z_{k-\frac{1}{2}}-z_{k+\frac{1}{2}} \text { for } k=1, \ldots, K .
\end{aligned}
$$

Also define $\Delta r_{i j k} \equiv \Delta x_{i} \Delta y_{j} \Delta z_{k}$. The $\left\{r_{i j k}\right\}$ are referred to as nodes (or grid points), and function values at these points are called nodal values. Assume that $\sigma$ and $\sigma_{s, n}$ have constant values on each cell

$$
\mathcal{Z}_{i j k} \equiv\left\{r \mid x_{i-\frac{1}{2}}<x<x_{i+\frac{1}{2}}, y_{j-\frac{1}{2}}<y<y_{j+\frac{1}{2}}, z_{k-\frac{1}{2}}<z<z_{k+\frac{1}{2}}\right\}
$$


denoted by $\sigma_{i j k}$ and $\sigma_{s, n}^{i j k}$, respectively. Function values that are constant on cells will be referred to as cell-centered values. We use $\psi_{i j k}$ to denote the approximation to $\psi\left(r_{i j k}\right)$, the true solution at $r_{i j k}$.

To obtain the finite volume method, average equation (2.3) over cell $\mathcal{Z}_{i j k}$. We then have a semi-discrete form

$$
\begin{aligned}
& \frac{\frac{1}{v} \partial \bar{\psi}_{i j k}}{\partial t}(\Omega, t)+\frac{1}{\Delta r_{i j k}} \int_{\mathcal{Z}_{i j k}} \Omega \cdot \nabla \psi(r, \Omega, t) d r+\bar{\sigma}_{i j k} \bar{\psi}_{i j k}(\Omega, t)= \\
& \sum_{n=0}^{\infty} \bar{\sigma}_{s, n}^{i j k} \sum_{m=-n}^{n} \bar{\phi}_{n, i j k}^{m}(t) Y_{n}^{m}(\Omega)+\bar{q}_{i j k}(\Omega, t),
\end{aligned}
$$

where the quantities marked with a bar are "cell-averaged" (in essence integral) quantities, and not point values. In particular:

$$
\begin{aligned}
& \bar{\psi}_{i, j, k}(\Omega, t)=\frac{1}{\Delta r_{i j k}} \int_{\mathcal{Z}_{i j k}} \psi(r, \Omega, t) d r, \\
& \bar{\phi}_{i, j, k}(t)=\frac{1}{\Delta r_{i j k}} \int_{\mathcal{Z}_{i j k}} \phi(r, t) d r \\
& \bar{q}_{i, j, k}(\Omega, t)=\frac{1}{\Delta r_{i j k}} \int_{\mathcal{Z}_{i j k}} q(r, \Omega, t) d r
\end{aligned}
$$

For the first integral in (3.6), use Green's Theorem to obtain

$$
\begin{aligned}
\frac{1}{\Delta r_{i j k}} \int_{\mathcal{Z}_{i j k}} \Omega \cdot \nabla \psi(r, \Omega, t) d r & =\frac{\mu}{\Delta x_{i}}\left(\bar{\psi}_{i+\frac{1}{2}, j, k}-\bar{\psi}_{i-\frac{1}{2}, j, k}\right) \\
& +\frac{\eta}{\Delta y_{j}}\left(\bar{\psi}_{i, j+\frac{1}{2}, k}-\bar{\psi}_{i, j-\frac{1}{2}, k}\right) \\
& +\frac{\xi}{\Delta z_{k}}\left(\bar{\psi}_{i, j, k+\frac{1}{2}}-\bar{\psi}_{i, j, k-1 \frac{1}{2}}\right)
\end{aligned}
$$

The averaged values with half-indexed subscripts now denote face values over each respective cell face. These too are cell-averaged values, but over faces instead of cells:

$$
\bar{\psi}_{i+\frac{1}{2}, j, k}=\frac{1}{\Delta y_{j} \Delta z_{k}} \int_{y_{j-\frac{1}{2}}}^{y_{j+\frac{1}{2}}} \int_{z_{k-\frac{1}{2}}}^{z_{k+\frac{1}{2}}} \psi\left(x_{i+\frac{1}{2}}, y, z, \Omega, t\right) d y d z
$$

and similarly for the other face values.

Note that with the substitution of (3.8) into (3.6) we have both cell-averaged and face-averaged quantities. To close the system, we express the face values in terms of the cell averages, often called the reconstruction procedure in the literature. This is the step where the spatial accuracy is determined in any finite volume scheme. For example, if we simply take

$$
\bar{\psi}_{i+\frac{1}{2}, j, k}=\left\{\begin{array}{l}
\bar{\psi}_{i, j, k}, \text { if } \mu>0 \\
\bar{\psi}_{i+1, j, k}, \text { if } \mu<0
\end{array}\right.
$$

we get the simple upwind, or "step" method. By replacing the face value by the average of the cell values on each side of the face we arrive at the diamond difference approximation:

$$
\bar{\psi}_{i+\frac{1}{2}, j, k}=\frac{1}{2}\left(\bar{\psi}_{i, j, k}+\bar{\psi}_{i+1, j, k}\right)
$$

If the interpolation scheme that relates the face- and cell-averages to each other is higher order, the spatial accuracy will be (at least formally) higher order as well. With linear interpolation schemes, however, oscillations will occur near discontinuities in the solution, in the source term, or in the material interfaces, or even at smooth, but steep gradients in the solution.

The WENO ("Weighted Essentially Nonoscillatory") method is in fact an interpolation scheme where the weights used in the linear combination of the interpolation points vary with the size of the derivative. The larger the gradient 
is near an interpolation point, the smaller the weight will be for that point. The idea goes back to the TVD (Total Variation Diminishing) method ([6]) which eliminated oscillations by choosing the left or right biased slope for reconstruction. The ENO (Essentially Nonoscillatory) [6] scheme raised the inherently second order accuracy of the TVD scheme by relaxing the TVD property to "essentially nonoscillatory" and choosing the interpolation stencil matching the order of accuracy from a set of available ones. With ENO the oscillations are not completely ruled out, but their size is reduced from $O(1)$ to $O\left(h^{r}\right)$, where $r$ is the order of accuracy. That is, in essence, oscillations were eliminated. The WENO scheme improved on this concept by not choosing, but rather, weighing the stencils by their smoothness.

3.3. The Weighted Essentially Nonoscillatory Interpolation. In this section we give a brief overview of the specific WENO method used in the numerical experiments. The formulae come mostly from [6], but for completeness we include them here. For clarity, we present the 1-D version of the interpolation, which can be readily used in a direction-by-direction fashion to reconstruct all three spatial dimensions in (3.8).

Given a cell averaged grid function $\left\{\bar{v}_{j}\right\}_{j=1}^{N}$ on a set of grid cells $\left\{x_{j}\right\}$ corresponding to a grid $\left\{x_{j-\frac{1}{2}}\right\}_{j=1}^{J}$, we approximate $v_{j+\frac{1}{2}}$ at the cell faces via a weighted linear combination of all possible interpolations:

$$
v_{j+\frac{1}{2}}=\sum_{r=0}^{k-1} w_{r} v_{j+\frac{1}{2}}^{(r)}
$$

where typically $k=2$ (for WENO3) or $k=3$ (for WENO5). The $v_{j+\frac{1}{2}}^{(r)}$ are the various interpolated values using polynomials corresponding to stencil $r$. The interpolation used for each stencil $r$ can, and in our code it does, take into account variable grid sizes, so no assumption of equally spaced grids is made.

The weights $w_{r}$ are given by:

$$
\begin{gathered}
w_{r}=\frac{\alpha_{r}}{\sum_{s=0}^{k-1} \alpha_{s}}, \quad \text { for } r=0, \ldots, k-1 \\
\alpha_{r}=\frac{d_{r}}{\left(\epsilon+\beta_{r}\right)^{2}}
\end{gathered}
$$

For the two most commonly used WENO schemes we have:

(i)if $k=2$ :

$$
\begin{aligned}
& d_{0}=\frac{2}{3} \\
& d_{1}=\frac{1}{3} \\
& \beta_{0}=\left(\bar{v}_{i+1}-\bar{v}_{i}\right) \\
& \beta_{1}=\left(\bar{v}_{i}-\bar{v}_{i-1}\right)
\end{aligned}
$$

(ii)if $k=3$ :

$$
\begin{aligned}
& d_{0}=\frac{3}{10} \\
& d_{1}=\frac{3}{5} \\
& d_{2}=\frac{1}{10} \\
& \beta_{0}=\frac{13}{12}\left(\bar{v}_{i}-2 \bar{v}_{i+1}+\bar{v}_{i+2}\right)^{2}+\frac{1}{4}\left(3 \bar{v}_{i}-4 \bar{v}_{i+1}+\bar{v}_{i+2}\right)^{2} \\
& \beta_{1}=\frac{13}{12}\left(\bar{v}_{i-1}-2 \bar{v}_{i}+\bar{v}_{i+1}\right)^{2}+\frac{1}{4}\left(\bar{v}_{i-1}-\bar{v}_{i+1}\right)^{2} \\
& \beta_{2}=\frac{13}{12}\left(\bar{v}_{i-2}-2 \bar{v}_{i-1}+\bar{v}_{i}\right)^{2}+\frac{1}{4}\left(\bar{v}_{i-2}-4 \bar{v}_{i-1}+3 \bar{v}_{i}\right)^{2}
\end{aligned}
$$


3.4. Boundary Conditions. For the boundary conditions in (2.4), when $x=x_{0}$, the normal $\vec{n}\left(r_{\frac{1}{2}, j, k}\right)=$ $(-1,0,0)$ for all $j, k$. Hence, $\vec{n}\left(r_{\frac{1}{2}, j, k}\right) \cdot \Omega=-\mu$, and for $\mu>0$ we have

$$
\bar{\psi}_{\frac{1}{2}, j, k}=g_{\frac{1}{2}, j, k}\left(\equiv g\left(r_{\frac{1}{2}, j, k}\right)\right) .
$$

For $y=y_{0}, \vec{n}\left(r_{i, \frac{1}{2}, k}\right)=(0,-1,0)$ for all $i, k$, and so $\vec{n}\left(r_{i, \frac{1}{2}, k}\right) \cdot \Omega=-\eta$, and for $\eta>0$ we have

$$
\bar{\psi}_{i, \frac{1}{2}, k}=g_{i, \frac{1}{2}, k}
$$

For $z=z_{0}, \vec{n}\left(r_{i, j, \frac{1}{2}}\right)=(0,0,-1)$ for all $i, j$, and so $\vec{n}\left(r_{i, j, \frac{1}{2}}\right) \cdot \Omega=-\xi$, and for $\xi>0$ we have

$$
\bar{\psi}_{i, j, \frac{1}{2}}=g_{i, j, \frac{1}{2}}
$$

The other three cases are handled similarly. Of course, for a given $\Omega=\Omega_{\ell}$ only three of the above six cases can hold. (The quadrature rules defined above guarantee that no component of $\Omega$ is ever zero.)

3.5. Temporal Discretization. The semidiscrete form (3.6) can be written as a matrix operation on a solution vector $\mathbf{\Psi}=\left(\psi_{i, j, k, l}\right)^{T}$ :

$$
\mathbf{V}^{-1} \dot{\Psi}+\mathbf{T} \mathbf{\Psi}-\mathbf{F}=0
$$

where: $\dot{\boldsymbol{\Psi}}=\frac{\partial \Psi}{\partial t}$ is the temporal derivative, $\mathbf{T}$ represents the semidiscretization in space and direction, and $\mathbf{F}$ includes source and boundary terms. In compact notation, we may write it as a system of ODE's. Note that the node centered version of the scheme would become a differential- algebraic equation (DAE) system

$$
F(t, \Psi, \dot{\Psi})=0
$$

because the boundary values would have to be solved for simultaneously with the interior values.

Since the code is general enough to have some node-centered spatial discretizations (such as Petrov-Galerkin), we use the same temporal solver for all spatial options. Hence for uniformity the time integration is accomplished via the IDA (Inexact Newton Differential/Algebraic Equation) package. It uses backward differencing methods which are variable in order (up to fifth order in time) and stepsize, and are also implicit.

4. Numerical Experiments. We now present several numerical results in 1-, 2-, and 3-D, using the same three-dimensional code, but simulating the lower dimensional problems by creating a very large (on the order of $10^{6}$ ) single grid cell in the irrelevant dimension. The initial condition in $(2.2)$ is $\psi^{0}(r, \Omega, E)=0$ for $t=0$ in all cases below. Also, the boundary conditions used were all Dirichlet BC and (2.4) was set via $g=0$ at all incident boundary faces.

4.1. 1-D Examples. We now present some time-dependent problems in 1-D slab geometry pointing out the salient features of the WENO method when compared to the more traditional, linear spatial discretizations.

(1) Single material, steady source. In this case the cross sections were set to $\sigma=0.1, \sigma_{s}=0.01$ with the source term defined by

$$
q= \begin{cases}10, & \text { if } x \in[0.4,0.6] \\ 0, & \text { otherwise }\end{cases}
$$

We used $M=50$ grid cells and compared WENO3 to the Petrov-Galerkin finite element (node centered) scheme and the first order upwind scheme of (3.9).

As it is illustrated on Figure 4.1, the third order WENO method can capture sharp transitions and corners significantly better than the first order accurate upwind method, while neither one produces oscillations. When compared to Petrov-Galerkin, it is clear that P-G creates large oscillations (and therefore negative fluxes) especially at the beginning stages of the development of the profile. This problem actually has a non-zero steady-state solution, to which the WENO3 method seems to converge faster than either of the other methods, as shown on the last figure 
of the series. In explaining this superior performance in convergence, we conjecture that the higher spatial accuracy is the explanation. Recall that the upwind method is first-, P-G is second-, and WENO3 is third-order accurate in smooth regions.

It is also interesting to compare the tested methods to a very fine grid upwind solution, which, in some sense, should be the most "reliable" in converging to the correct physical solution under grid refinement. On Figure 4.2 we show the temporal behavior of a single point located at $x=0.6$ and include a 1000 cell upwind solution for reference. Note how the WENO is extremely close to the "super-fine" upwind profile, while the other two methods are off by about $10 \%$.

(2) Single material, steady point-source. In order to test the method for a very narrowly supported pseudo pointsource, we ran the same problem as in case (1) above, but with a source defined as

$$
q= \begin{cases}10, & \text { if } x \in[0.48,0.52] \\ 0, & \text { otherwise }\end{cases}
$$

where now the source is nonzero in only two grid cells. This is a challenge for spatial discretizations which are higher than first order, because the jumps caused by the source are closer to each other than the stencil width itself. We now included WENO5 in the comparison to push the limits of the scheme for the WENO5 scheme uses a stencil that is 5 cells wide.

As shown on Figures 4.3 and 4.4, the behavior of the WENO schemes is still acceptable. They produce no oscillations at the base of the source, but they both overshoot the "best" solution, the super-fine upwind. The expectation is that as the grid is refined the behavior will be identical to that of the previous case, since the distance between locations of the large gradients will then be several grid cells wide.

(3) Thin/thick materials, large unsteady source. We now test the code on a two-material problem defined by:

$$
\sigma(x)= \begin{cases}0.0001, & \text { if } x \in[0,0.1] \\ 100, & \text { otherwise }\end{cases}
$$

and the source, localized by $x \in[0,0.1]$, changes in time as:

$$
q(t)= \begin{cases}8 \times 10^{12}, & \text { if } t \in\left[10^{-9}, 4 \times 10^{-9}\right] \\ 0, & \text { otherwise }\end{cases}
$$

The suite of methods tested now includes not just WENO3 and WENO5, upwing, and P-G, but the Simple Corner Balance (SCB) and the Diamond-Difference with negative flux fix-up (D-D) methods as well; both of the latter are cell-centered schemes. We compare all these methods on a grid of $M=200$ cells with a very fine grid upwind solution on 5000 cells. The latter is again guaranteed to converge to the right solution under grid refinement.

An examination of Figure 4.5 reveals the two salient features of the WENO scheme: (i) its nonoscillatory nature and (ii) high order of accuracy in smooth regions. The latter property is more pronounced when the solution has areas of smooth variation. In Figure 4.5 we show close-ups of the highly varying regions on the right side of each snapshot. These confirm our expectation of the WENO scheme coming very close to the superfine solution, while the lower order schemes produce large errors near extrema and/or oscillations near steep gradients. To avoid having to include a large number of plots, we chose to use the integrated quantity $p h i$ in Figure 4.5

(4) Thick/thin materials, large unsteady source. If we reverse the two material properties, but leave the source term as in Case (3), we can test the code on a "thick-to-thin" problem. Thus set:

$$
\sigma(x)= \begin{cases}100, & \text { if } x \in[0,0.1] \\ 0.0001 . & \text { otherwise }\end{cases}
$$

In this case the high order of accuracy offered by the WENO method is quite pronounced near the corner regions of the profiles. The WENO3 and WENO5 schemes seem to give the same accuracy as the upwind scheme on a very fine grid - but with $1 / 25$ th of the number of cells. 
4.2. 2-D Test Problem. The method is now tested in $2-\mathrm{D}$ mode using the same code, but now the (single) cell in the $z$-direction is very large to simulate a 2-D slab. Initial and boundary conditions are again set to be nil, and the grid is a $300 \times 300$ Cartesian mesh in a $[0,100] \times[0,100]$ square domain. Cross sections were taken to be $\sigma=0.001$ and $\sigma_{s}=0.001$, and the source was defined by:

$$
q= \begin{cases}1, & \text { if }(x, y) \in[33.25,66.75] \times[33.25,66.75] \\ 0, & \text { otherwise }\end{cases}
$$

This was actually a steady-state simulation where we used the KINSOL package as our nonlinear solver. The same results were arrived at running the unsteady option out to steady-state.

On the top portion of Figure 4.7 we show a contour plot of the flux when both direction are positive. We also ran the upwind and SCB methods for comparison. The latter showed some oscillations and severe inaccuracy in capturing the middle part of the profile, even at steady-state. The upwind method, due to its dissipative nature, "smoothed" out the corners. Both WENO3 and WENO5 performed well, but except for the sharp left corner, there was little difference between the two.

4.3. 3-D Test Problem. We finally ran the code in fully $3-D$ mode, on a domain $[0,1] \times[0,1] \times[0,1]$ with $40 \times 40 \times 40$ grid cells. Cartesian mesh in a $[0,100] \times[0,100]$ square domain. In this case we set $\sigma=10.0$ and $\sigma_{s}=0$. The source term was again in the middle:

$$
q= \begin{cases}1, & \text { if }(x, y, z) \in[.4, .6] \times[.4, .6] \times[.4, .6] \\ 0, & \text { otherwise }\end{cases}
$$

This case was again run in steady-state mode and then verified by the unsteady option.

On Figure 4.8 we show the two WENO options, Petrov-Galerkin and upwind on two different grids $(40 \times 40 \times 40$ and $100 \times 100 \times 100$ ) at the middle cut of $y=0.5, z=0.5$. The finer upwind solution was not run on a grid that is orders of magnitude finer than the others, hence it should not be taken now as a benchmark solution. It is included merely to show the difference grid refinement makes in the accuracy of the solution. The WENO3 and WENO5 solutions are expected to be better, and in fact they are somewhat different from each other as well. The Petrov-Galerkin method, on the other hand, exhibits sizeable oscillations/negative fluxes near the profile corners.

5. Discussion. We have presented a new application of the WENO scheme for the spatial discretization of the Boltzmann Transport Equation. Developed for highly nonlinear systems of partial differential equations, such as the Euler and Navier-Stokes equations, the scheme was designed to accurately compute shocked fluid flow. As such, it was an unlikely candidate for application to a scalar and linear integro-differential equation. Indeed, the notion of "slope-limiting," and in general, of nonlinear schemes has been known for over two decades, yet unutilized (to our knowledge) in the transport arena. However, once the realization is made that it is merely an interpolation technique, it is a natural fit for any problem where the problem is linear, but the coefficients or the source terms are discontinuous and thus give rise to steep gradients in the solution.

We hope to have demonstrated that the WENO scheme can be very useful for many problems, especially unsteady ones. While they do not guarantee positivity, by reducing the size of oscillations to $O\left(h^{r}\right)$, in practice they give positive fluxes in an overwhelming majority of the cases where other linear schemes fail to do so. We have shown that the high order of spatial accuracy on a relatively coarse grid can be interchangeable with a very fine grid spacing using a low order method. This translates into an often sought-after, and now possible trade-off between processor power and memory capacity. The WENO method seems to be a good choice especially for cases where the solution simultaneously has steep gradients/discontinuities and large areas of smooth variations.

For future work, we intend to further improve on the essentially nonoscillatory property to ensure positivity by lowering the order of accuracy in those rare regions where small negative fluxes still remain. We also plan to develop better sweep-preconditioners that mimic the behavior of the nonlinear discretization used in the WENO scheme itself. Furthermore we need to conduct rigorous grid refinement studies to verify the order of accuracy at least in the ideal situation where the source term is smooth and an exact solution exists (no scattering). Finally, more testing is necessary on large 3-D problems with multiple material and different source terms. 
Acknowledgements. The authors wish to acknowledge IPAM (Institute for Pure and Applied Mathematics) at UCLA for co-sponsoring the first (summer) project in this subject area. In particular we thank Richard Tsai, Filip Matejka, David Stevens, Nicholas Kridler and Rodney Chan for their initial contributions in summer of 2002.

References. [1] V. Faber and T. A. Manteuffel, A Look at Transport Theory from the Point of View of Linear Algebra, Transport Theory, Invariant Imbedding, and Integral Equations,P. Nelson et al, eds.,Marcel Dekker, New York,1989,pp 37-61.

[2] G. C. Pomraning, The Equations of Radiation Hydrodynamics, Pergamon Press, Oxford, 1973.

[3] R. L. Liboff,Introductory Quantum Mechanics, Holden-Day, Inc.,San Francisco,1980.

[4] S. F. Ashby, P. N. Brown, M. R. Dorr and A. C. Hindmarsh, Preconditioned Iterative Methods for Discretized Transport Equations, UCRL-JC-104901, Lawrence Livermore National Laboratory,1990.

[5] B. G. Carlson and K. D. Lathrop, Transport Theory: The Method of Discrete Ordinates, Computing Methods in Reactor Physics, H. Greenspan et al. eds.,Gordon and Breach, New York,1968,pp.166-266.

[6] C-W Shu, Essentially Non-Oscillatory and Weighted Essentially Non-Oscillatory Schemes for Hyperbolic Conservation Laws, ICASE Report No. 97-65, NASA Langley Research Center, 1997.

This work was performed under the auspices of the U. S. Department of Energy by University of California, Lawrence Livermore National Laboratory under contract No. W-7405-Eng-48. 

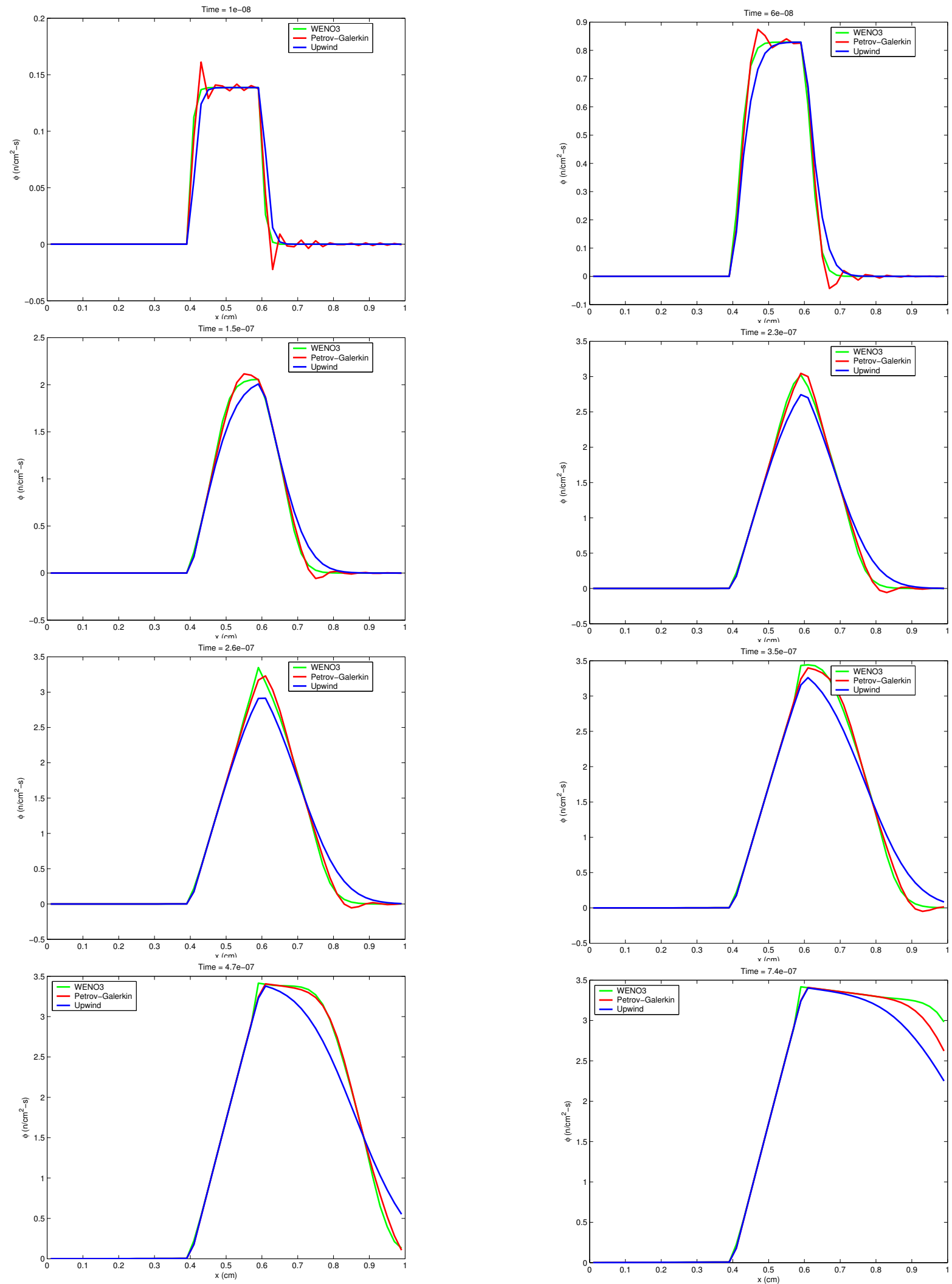

FIG. 4.1. Comparison of the WENO, $P-G$, and upwind methods for $\mu>0$ at different times 


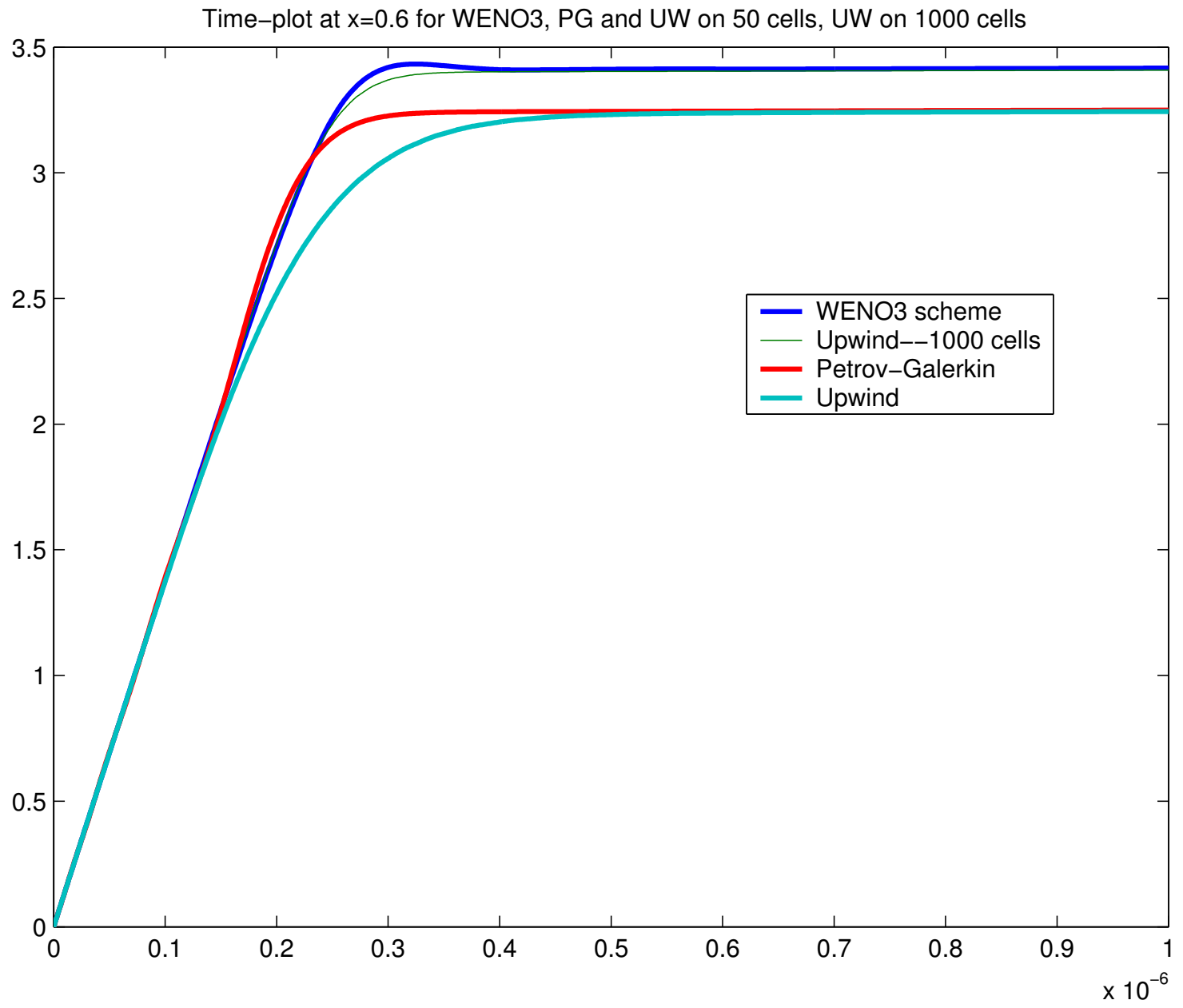

FIG. 4.2. Time evolution of the solution at $x=0.6$ 

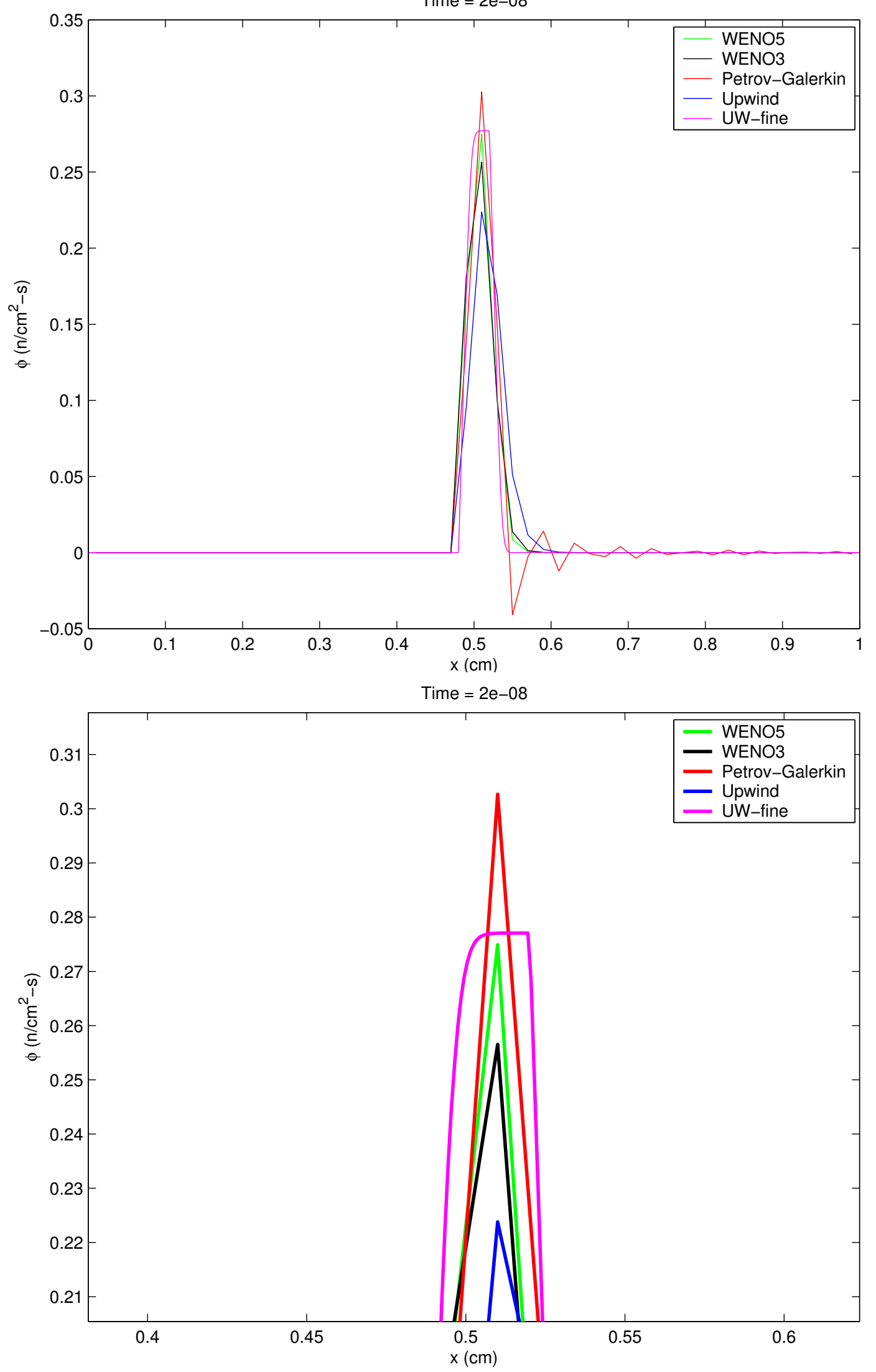

13

FIG. 4.3. Comparison of the WENO, $P-G$, and upwind (coarse and fine grid) methods for $\mu>0$ at $t=2 \times 10^{-8}$ (bottom fig.is close-up) 


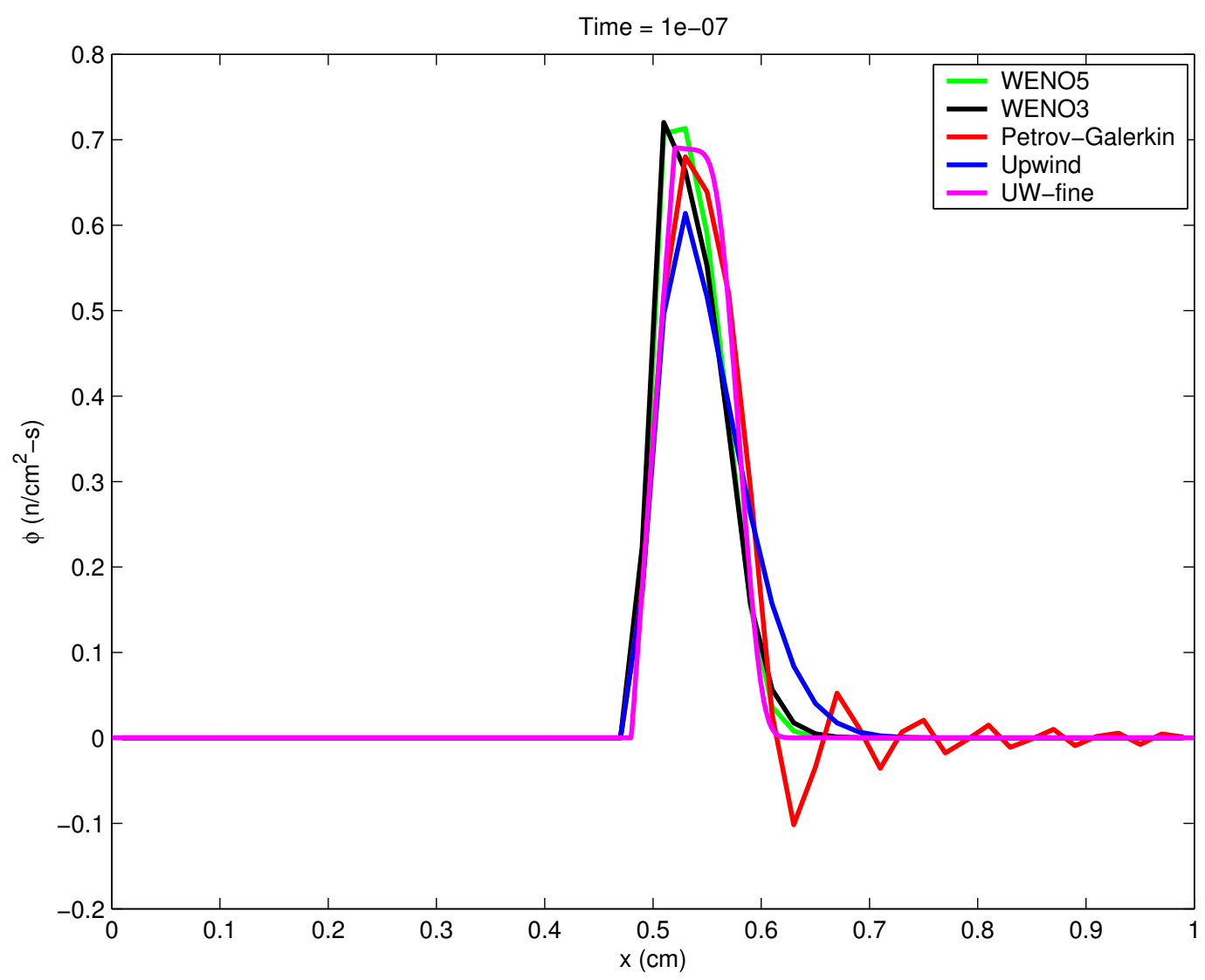

FIG. 4.4. Comparison of the WENO, $P$-G, and upwind (coarse and fine grid) methods for $\mu>0$ at $t=10^{-7}$ 

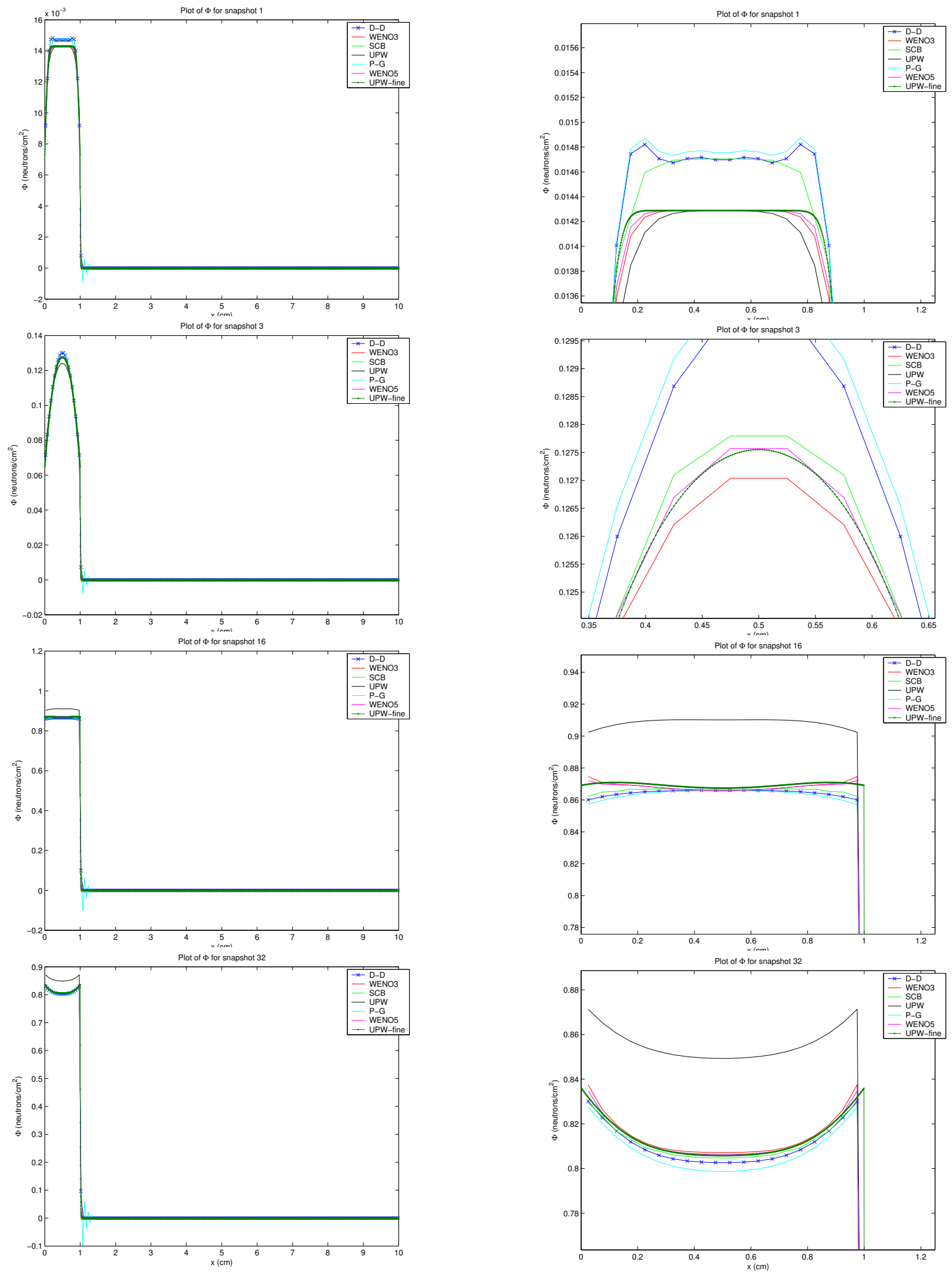

FIG. 4.5. Comparison of the WENO, P-G,SCB, and upwind methods with the fine-grid upwind for the thin-tothick problem. 

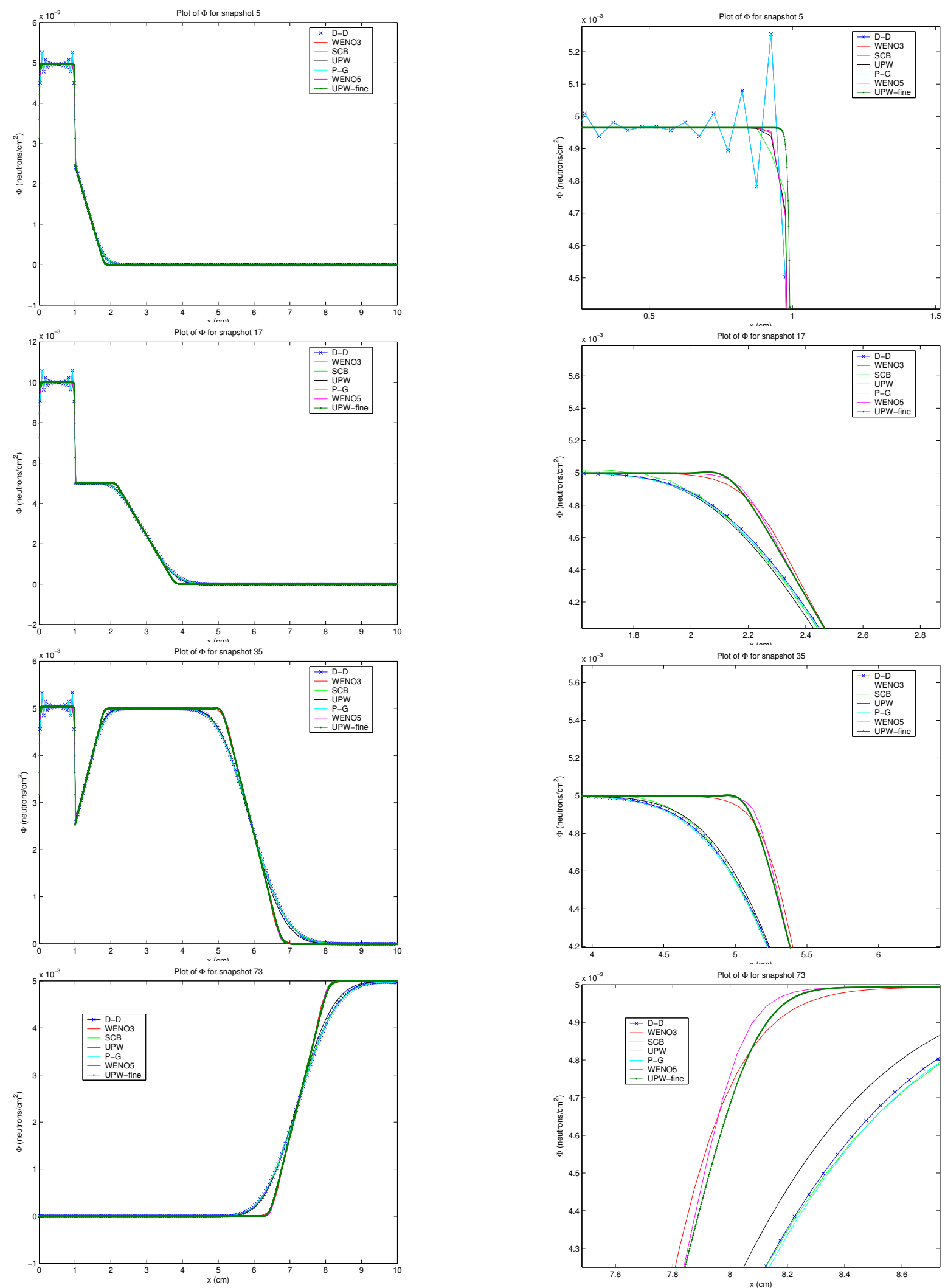

FIG. 4.6. Comparison of the WENO, $P-G, S C B$, and upwind methods with the fine-grid upwind for the thich-tothin problem. 


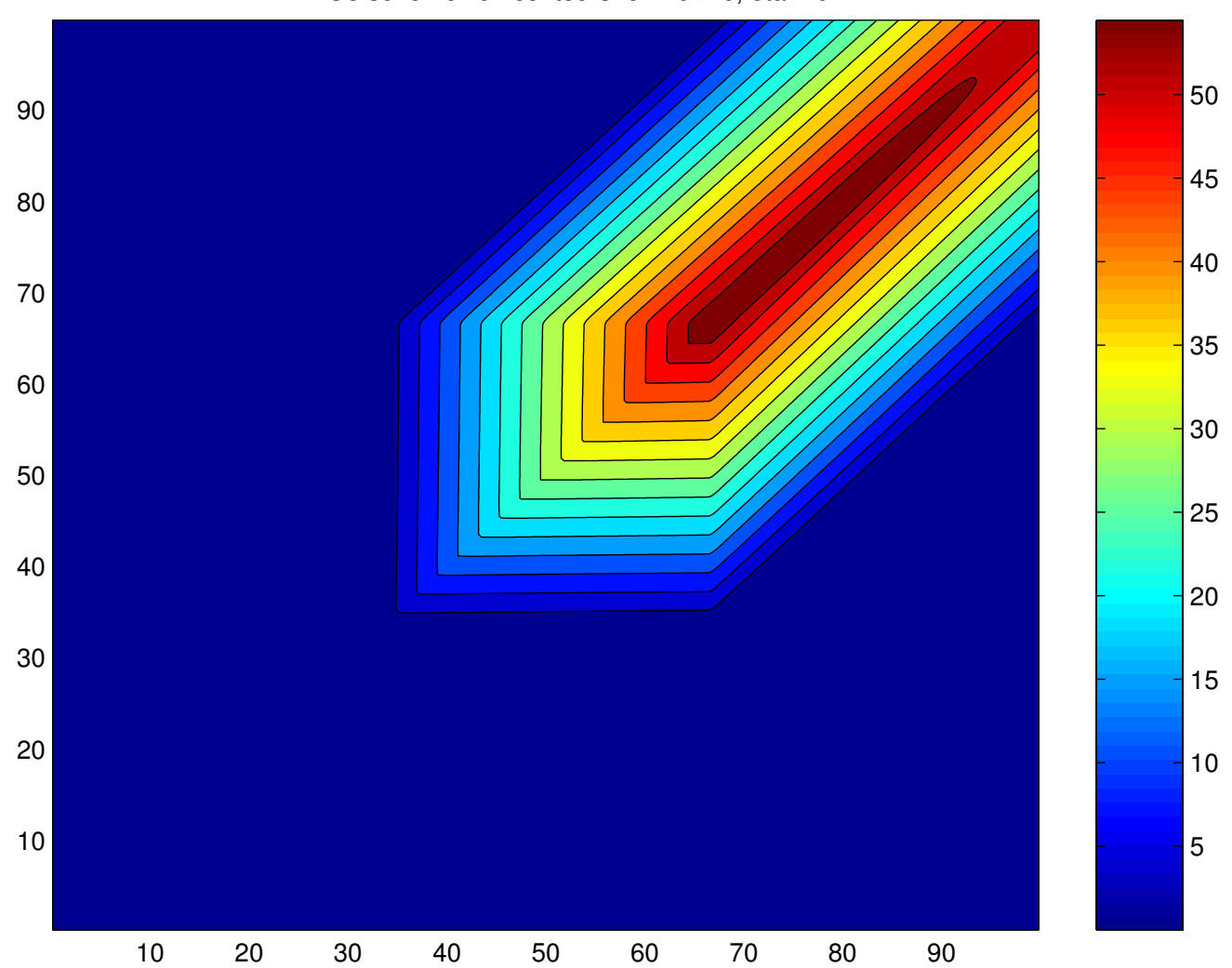

Flux at $\mathrm{j}=105$ for WENO5,WENO3, Corner balance, and Upwind schemes

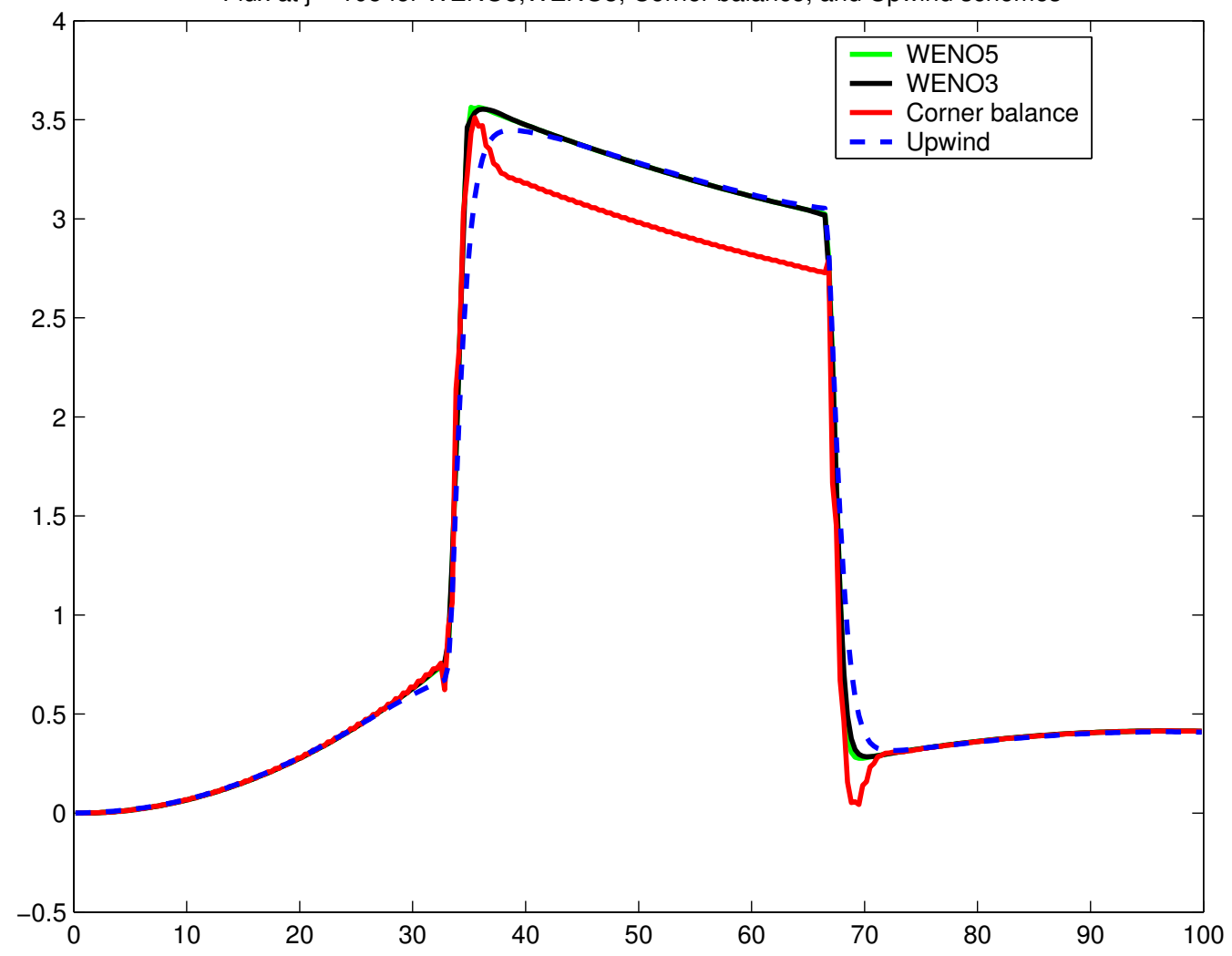


Particle flux for $\mu>0$, at $y=0.5, z=0.5$, with $N_{x}=40$

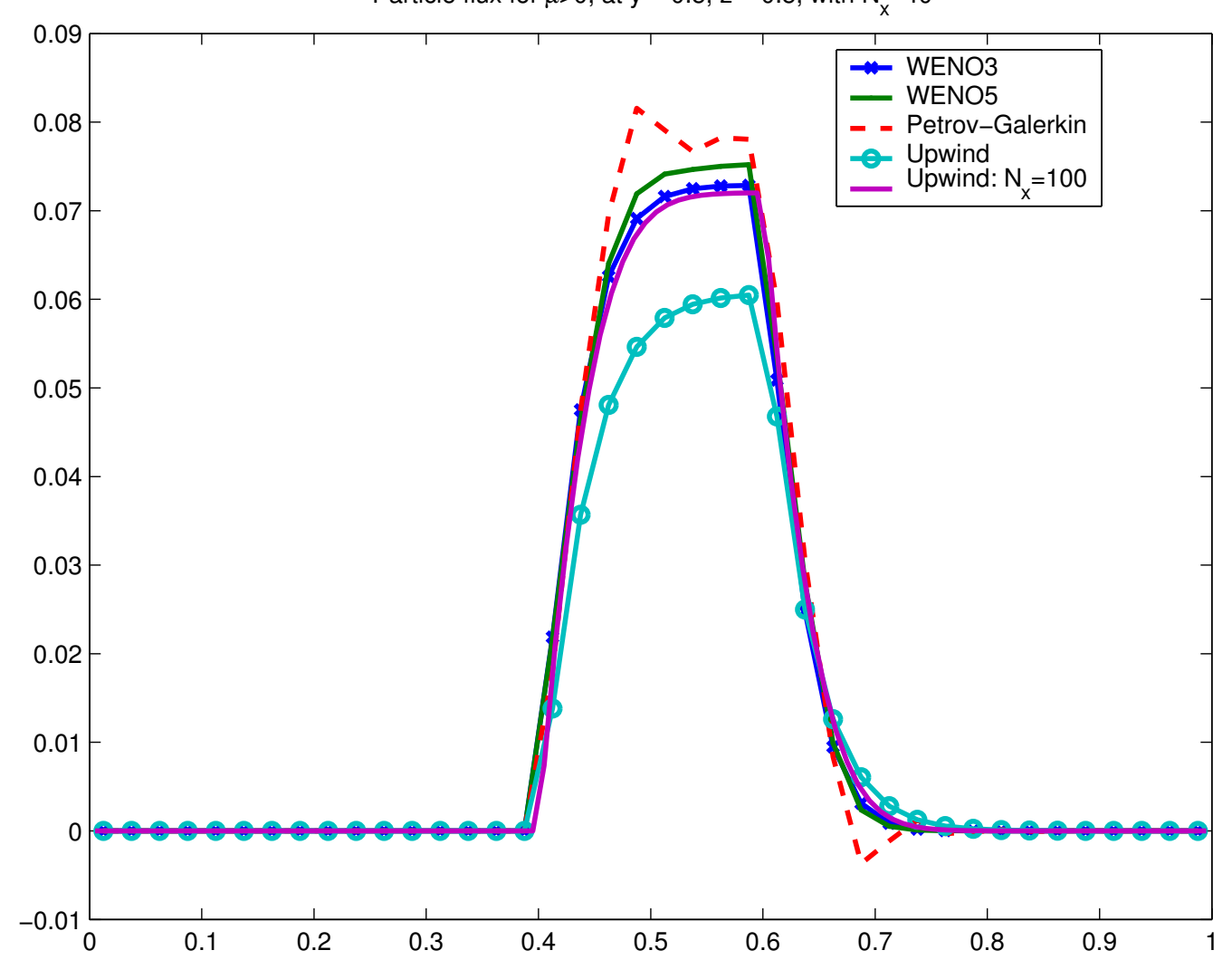

FIG. 4.8. Comparison of the WENO, P-G, and upwind methods for the 3-D test case 